\title{
Materials, Designs, Fabrications, and Applications of Organic Electronic Devices
}

\author{
Jwo-Huei Jou, ${ }^{1}$ Ramunas Lygaitis, ${ }^{2}$ K. R. Justin Thomas, ${ }^{3}$ and Liang-Sheng Liao ${ }^{4}$ \\ ${ }^{1}$ Department of Material Science and Engineering, National Tsing Hua University, Hsinchu 30013, Taiwan \\ ${ }^{2}$ Department of Polymer Chemistry and Technology, Kaunas University of Technology, Radvilenu Road 19, 50254 Kaunas, Lithuania \\ ${ }^{3}$ Organic Materials Laboratory, Department of Chemistry, Indian Institute of Technology, Roorkee 247667, India \\ ${ }^{4}$ Institute of Functional Nano and Soft Materials, Soochow University, Suzhou 215123, China
}

Correspondence should be addressed to Jwo-Huei Jou; jjou@mx.nthu.edu.tw

Received 4 August 2014; Accepted 4 August 2014; Published 14 August 2014

Copyright (C) 2014 Jwo-Huei Jou et al. This is an open access article distributed under the Creative Commons Attribution License, which permits unrestricted use, distribution, and reproduction in any medium, provided the original work is properly cited.

Organic electronic devices (OEDs) are pronounced combination of sustainable organic materials, easily processable, potentially low cost, high throughput, and large-area rollto-roll fabrication techniques that open up the numerous new fields of applications. The estimated global revenue was $\$ 16$ billion for OEDs in 2013 and is expected to reach \$77 billion in 2023 [1]. According to projected revenue reports $[1,2]$, organic light emitting diodes (OLEDs) and organic photovoltaics (OPVs) contribution is to be over $50 \%$, which clearly states that both OLEDs [3] and OPVs [4] will play a major role in OEDs growth. Nowadays, OED technology is on the boom of reliability for numerous cutting edge applications. OEDs are drastically increasing the usage in highquality OLED based displays and lightings, OPVs, smart packaging, medical products, radio-frequency identity tags, organic memory devices, organic batteries, organic sensors, and so forth.

Amongst, OLED based displays have attracted the greatest attention owing to their numerous disruptive features, such as energy efficiency, potentially low cost, light weight, ultrathin, wide viewing angle, fast response time, and ultrahigh contrast. OLED devices can be fabricated either by solution process or by thermal evaporation. Wet-process enables the formation of cost-effective large-size roll-toroll fabrication, while dry-process possesses the formation of well-controlled, high-quality films with ultimate design freedom for devices. However, both techniques have some severe issues, which must be resolved sooner to realize a commercially competitive display or lighting device. In their paper, F.-C. Tung et al. developed a large-area feasible novel thin-film deposition technique with a planar source loadable with any premetered solvent mixed organic compounds, plausibly with no component number limitation. The innovative technology would offer an over $70 \%$ material utilization rate with ultimately high thin layer uniformity ( $95 \%)$. In order to achieve high efficiency and long operation lifetime in OLEDs, S.-W. Liu et al. investigated the role of deposition rate during thermal evaporation in high vacuum. Optimization of the deposition rate was applied to control the layer interface by molecular packing of planar molecule, such as bis(10-hydroxybenzo[h] qinolinato)beryllium, which can effectively reduce the driving voltage and increase the device efficiency as well as operating lifetime. For example, the operating lifetime of the two-step deposition OLED was 4.6 times longer than that with a single deposition rate.

At the present time, active-matrix OLED (AM-OLED) is an extensively acceptable display technology for portable electronic devices and is swiftly penetrating in large-area displays. In C.-L. Fan et al.'s paper, the authors proposed a new pixel circuit design, which comprises five transistors and one capacitor to supply the uniform current, and driving method to enhance the brightness uniformity of AM-OLEDs. They have employed the automatic integrated circuit modelling simulation programme with integrated circuit emphasis simulator for the voltage programming method of the proposed pixel circuits. Y.-S. Tsai et al. demonstrated a synthetic 
graphite fiber as the heat dissipation substrate for topemission OLED to reduce the joule heating. High dissipation graphite substrate extensively reduces the substrate heating rate to $0.037^{\circ} \mathrm{C} / \mathrm{sec}$ and realized a lifetime of 1.875 times higher than that of typical glass substrate.

OLEDs are steadily penetrating in lighting industry owing to their several unique characteristics, like very high color rendering index, sunlight style emission [5], candle light style emission [6,7], physiologically friendly energy saving, and potentially cost-effective. Color temperature of light plays a crucial role in regulating human's circadian rhythm. It is well known that current lighting sources provide only a fixed color temperature light emission. Hence, devising a cost-effective light source with color temperature tunability, which can cover the entire daylight locus, would be highly required. S.-Y. Liao et al. reported a cost-effective sunlight style OLED device with a wide color temperature span of $7,000 \mathrm{~K}$. They have also reported that the color temperature span can be made much wider without any additional carrier modulation layer. In order to realize a low cost and simple flexible OLED lighting, C.-C. Chen et al. reported a warm white OLED device from blue OLED by using a yellow phosphor embedded polydimethylsiloxane (PDMS) film on glass substrate. The resultant OLED device has shown the chromaticity coordinates of 0.38 and 0.54 and color temperature of $4,200 \mathrm{~K}$.

The global demand for energy is increasing very fast, whereas the fossil (petroleum and coal) energy resources are draining rapidly. OPV is one future technology with enormous potential to supply an alternative clean energy source, which is projected to reach a higher profitability at a lower level cost of electricity. In recent years, OPVs have gained widespread attention due to their numerous superlative features, such as low cost, large area, flexible, roll-to-roll fabrication, and tunable optical properties. H. Ninsonti et al. synthesized the gold loaded titanium dioxide (Au-loaded $\mathrm{TiO}_{2}$ ) nanoparticles by the sol-gel technique to enhance the performance of dye-sensitized solar cells (DSSC). It is found that the $1.0 \mathrm{~mol} \% \mathrm{Au}$-loaded $\mathrm{TiO}_{2}$ possesses the plasmon effect and scattering property. In their paper, H.-Y. Lee and H.-L. Huang reported an inverted polymer solar cell (PSC) by employing pentacene-doped P3HT:PCBM absorption layers. They have found that the power conversion efficiency of inverted pentacene-doped PSCs is over 27\% higher than that of PSCs without the pentacene doping. In future, the recycling of solar-cell silicon wafers is highly demanded. P. S. $\mathrm{Pa}$ reported in their paper that an ultrasonic electrochemical micromachining with high magnetic strength has facilitated a large discharge effect and fast removal of epoxy and silicon nitride hybrid composite layer. It is found that this newly developed technique would lead to an easy recycling of defective or used silicon wafers in the OPV industries.

All these research articles present a pioneering observation into the state of the art, as well as promising prospect for next generation displays, lightings, and renewable green energy sources. As reported in respective research articles, the cost-effective, high performance mass production of OLEDs and OPVs can easily be enhanced by the novel materials synthesis, tailoring of device architectures, development of new fabrication technologies, and materials recycling tools. This special issue is going to contribute significantly to the development and commercialization of OLEDs and OPVs.

Jwo-Huei Jou

Ramunas Lygaitis

K. R. Justin Thomas

Liang-Sheng Liao

\section{References}

[1] R. Das and P. Harrop, "Printed, organic and flexible electronics: forecasts, players and opportunities 2013-2023," http://www idtechex.com/research/reports/printed-organic-and-flexibleelectronics-forecasts-players-and-opportunities-2013-2023000350.asp? viewopt $=$ desc.

[2] http://www.transparencymarketresearch.com/global-organicelectronics-market.html.

[3] M. C. Gather, A. Köhnen, and K. Meerholz, "White organic light-emitting diodes," Advanced Materials, vol. 23, no. 2, pp. 233-248, 2011.

[4] W. Cao and J. Xue, "Recent progress in organic photovoltaics : device architecture and optical design," Energy \& Environmental Science, vol. 7, pp. 2123-2144, 2014.

[5] J.-H. Jou, M.-H. Wu, S.-M. Shen et al., "Sunlight-style colortemperature tunable organic light-emitting diode," Applied Physics Letters, vol. 95, no. 1, Article ID 013307, 2009.

[6] J.-H. Jou, C.-Y. Hsieh, J.-R. Tseng et al., "Candle light-style organic light-emitting diodes," Advanced Functional Materials, vol. 23, no. 21, pp. 2750-2757, 2013.

[7] J.-H. Jou, P.-W. Chen, and Y.-L. Chen, "OLEDs with chromaticity tunable between dusk-hue and candle-light," Organic Electronics, vol. 14, no. 1, pp. 47-54, 2013. 

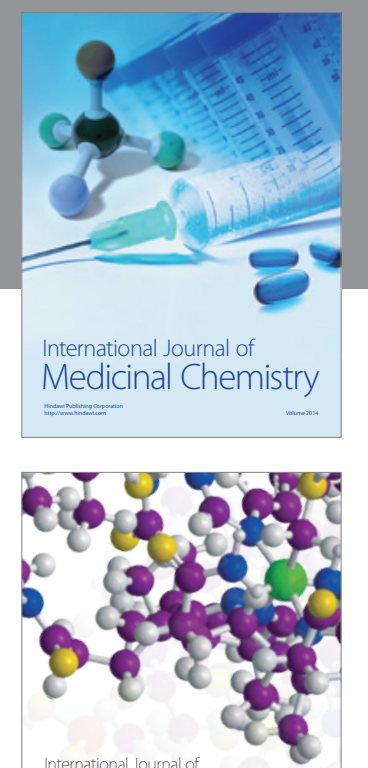

\section{Carbohydrate} Chemistry

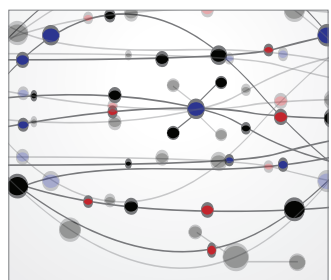

The Scientific World Journal
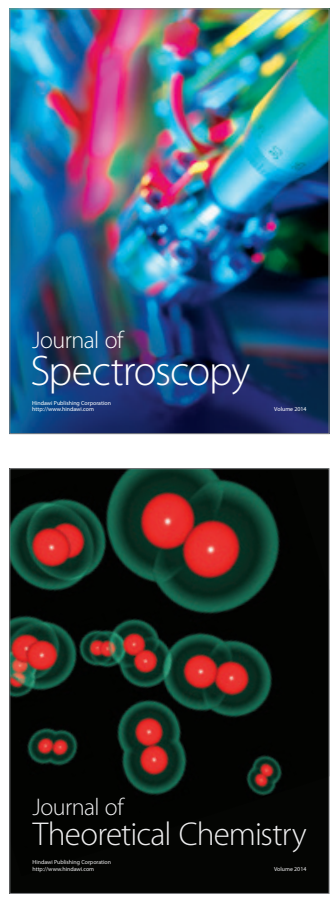
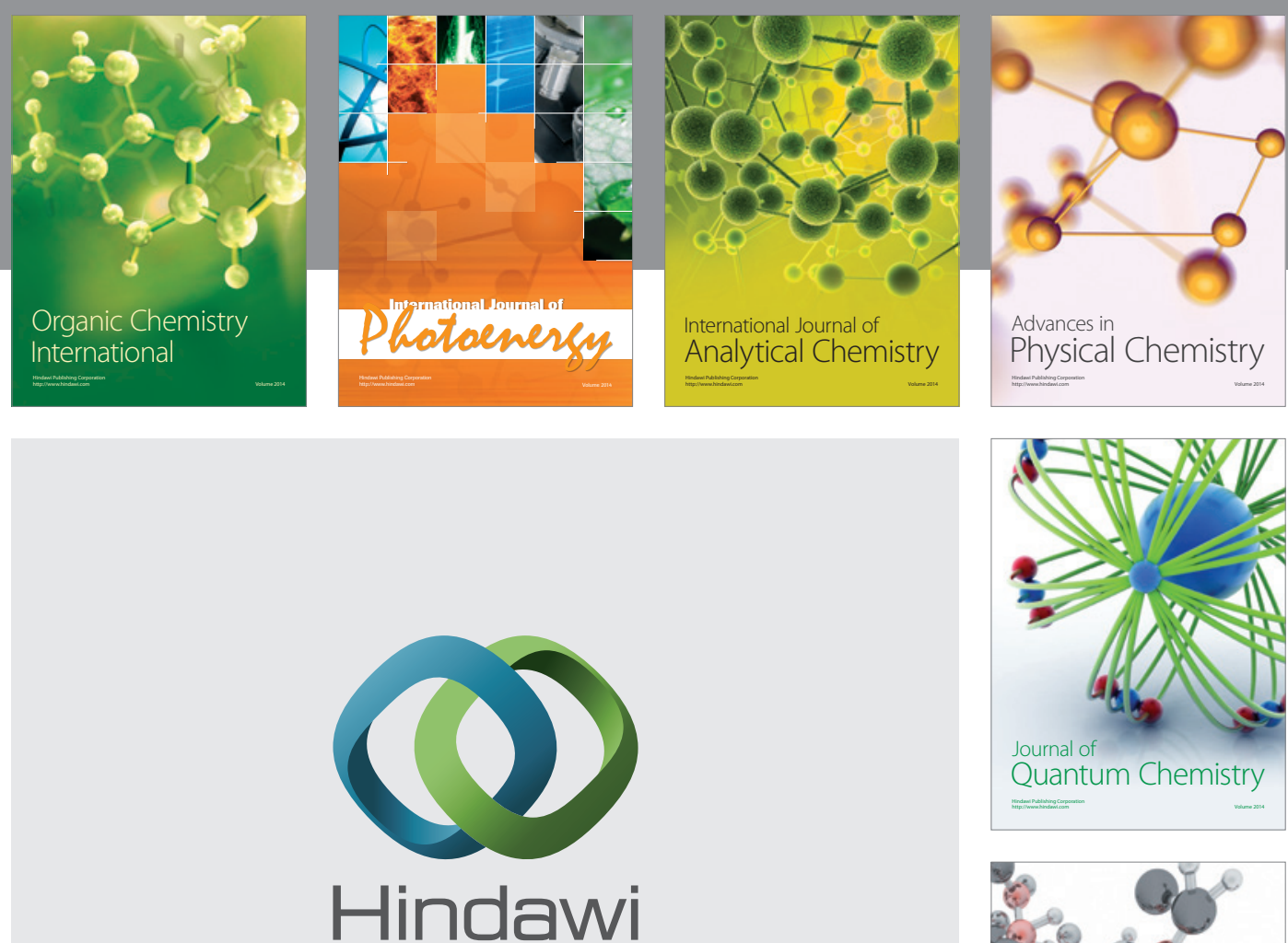

Submit your manuscripts at

http://www.hindawi.com

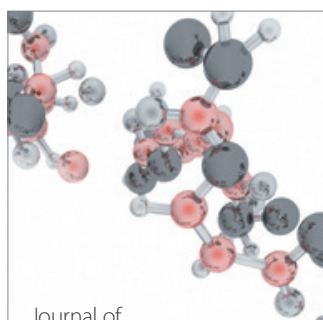

Analytical Methods

in Chemistry

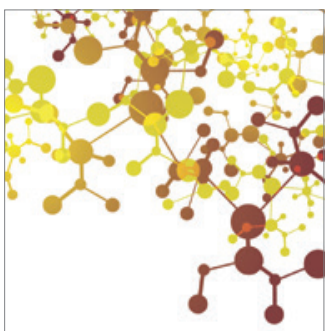

Journal of

Applied Chemistry

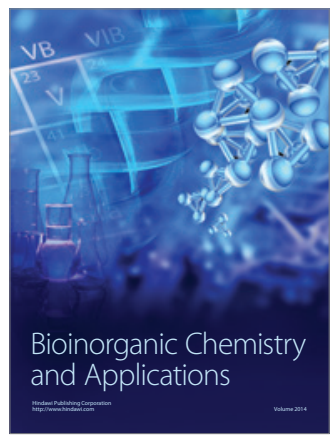

Inorganic Chemistry
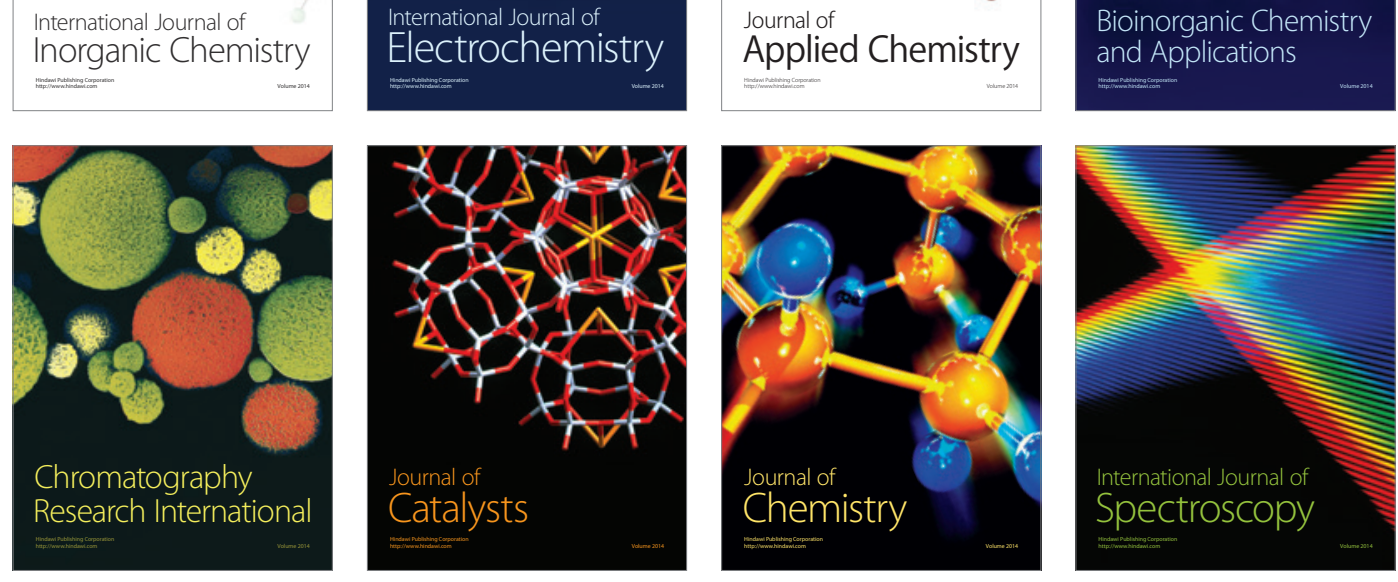